\title{
Sensitization of Dorsal Horn Neurons in a Two-Compartment Cell Culture Model: Wind-Up and Long-Term Potentiation- Like Responses
}

\author{
Kristina S. Vikman, Krister Kristensson, and Russell H. Hill \\ Department of Neuroscience, Karolinska Institutet, SE-171 77 Stockholm, Sweden
}

One of the main characteristics of central sensitization associated with postinjury pain and chronic pain is increased excitability of the dorsal horn neurons in the spinal cord. Two electrophysiological features associated with the origin and modulation of central sensitization are wind-up of action potential frequency and long-term potentiation (LTP), which have been demonstrated previously in the intact dorsal horn. Here we present evidence for electrically evoked sensitization of dorsal horn neurons in a two-compartment cell culture system of rat dorsal root ganglia (DRGs) and dorsal horn neurons. Whole-cell recordings of dorsal horn neurons showed that repetitive low-frequency stimulation of DRG axons induced a frequency-dependent cumulative depolarization of the membrane potential with a concomitant increase in action potential

Sensitization at the level of the spinal cord is a component of postinjury pain hypersensitivity, because it has been shown that increased sensitivity after injury is not only attributable to peripheral sensitization (e.g., reduction in threshold of nociceptors) but is also a consequence of increased excitability of dorsal horn neurons (Woolf, 1983). Central sensitization in the dorsal horn of the spinal cord is characterized by increased spontaneous activity, enlarged receptive field areas, and increased responses evoked by large- and small-caliber primary afferent fibers (Woolf, 1996). Two phenomena described in connection with central sensitization, and which may be implicated in its generation, are wind-up (Mendell and Wall, 1965) and long-term potentiation (LTP) (Randic et al., 1993; Liu and Sandkühler, 1997; Svendsen et al., 1997). The former is characterized by a progressive, low frequency-dependent increase in excitability of dorsal horn neurons produced by constant, repetitive $\mathrm{C}$-fiber stimulation and is of relatively short duration, whereas LTP, which can be elicited by high-frequency stimulation (HFS), is of longer duration. Although the cellular mechanisms of central sensitization and its relation to postinjury pain hypersensitivity and hyperalgesia are still not fully elucidated, the two phenomena, wind-up and LTP, have become important tools for studies of factors that are involved in the generation and modulation of spinal sensitization (for review, see Baranauskas and Nistri, 1998; Herrero et al., 2000).

\footnotetext{
Received May 1, 2001; revised July 2, 2001; accepted July 11, 2001.

This study was supported by grants from the Swedish Medical Research Council, project 4480.

Correspondence should be addressed to Kristina S. Vikman, Department of Neuroscience, B2:5, Karolinska Institutet, Retzius väg 8, SE-171 77 Stockholm, Sweden. E-mail: kristina.vikman@neuro.ki.se.

Copyright (ㄷ) 2001 Society for Neuroscience $0270-6474 / 01 / 210001-06 \$ 15.00 / 0$
}

frequency in a subset of neurons (41\%). The characteristics presented here for dissociated cells are in accordance with those ascribed to classical wind-up in the intact dorsal horn. In addition, tetanic stimulation of DRG axons resulted in a significant increase in the number of action potentials in response to test stimuli in $42 \%$ of the cells tested. This prolonged potentiation of neuronal excitability in the dorsal horn lasted throughout the recording period $(>1 \mathrm{hr}$ ) and tended to be voltage dependent in an LTP-like manner. To our knowledge, this is the first time that wind-up and LTP-like responses are reported for dorsal horn neurons in cell culture.

Key words: DRG; nociception; pain; patch clamp; spinal cord; stimulus-evoked potentials; synaptic plasticity

The majority of studies on wind-up and LTP in the dorsal horn have been performed on intact preparations or slices of spinal cord. These techniques maintain the anatomical structure of the spinal cord but are of limited use for detailed studies of modulation and interaction at the synaptic level. Recently, we have described a two-compartment cell culture model based on DRG and dorsal horn neurons in which the presynaptic DRG neurons and the target dorsal horn neurons are separated by a diffusion barrier but are in synaptic contact with each other (Vikman et al., 2001). In this model, the DRG axons in one compartment can be selectively stimulated while postsynaptic events are recorded in the dorsal horn neuronal network in the other compartment. We also demonstrated that a subpopulation of the DRG neurons in the system possesses characteristics of $\mathrm{A} \delta$ - and $\mathrm{C}$-fiber neurons (i.e., nociceptive neurons), such as responsiveness to capsaicin, calcitonin gene-related peptide immunoreactivity, and tetrodotoxin-resistant sodium channels.

The present study was designed to investigate whether the synaptic transmission between DRG and dorsal horn neurons in

This article is published in The Journal of Neuroscience, Rapid Communications Section, which publishes brief, peerreviewed papers online, not in print. Rapid Communications are posted online approximately one month earlier than they would appear if printed. They are listed in the Table of Contents of the next open issue of JNeurosci. Cite this article as: JNeurosci, 2001, 21:RC169 (1-6). The publication date is the date of posting online at www.jneurosci.org.

http://www.jneurosci.org/cgi/content/full/5650 
the system exhibits electrophysiological features characteristic of central sensitization. Here we present evidence that both lowfrequency stimulation (LFS) and HFS of DRG neurons induce increases in the neuronal responsiveness of dorsal horn neurons in this two-compartment model. The results are in accordance with those observed previously in intact spinal cord and slice preparations, and this is, to our knowledge, the first time these phenomena are described in cell culture. These findings indicate that this model is suitable as an efficient tool to analyze the mechanisms of sensitization of dorsal horn neurons at the cellular level and how it may be modulated by factors that affect nociception.

\section{MATERIALS AND METHODS}

Two-compartment cultures. Procedures for establishment of twocompartment culture dishes and dissection of tissue, as well as the composition of the culture medium, have been described in detail by Vikman et al. (2001). Briefly, two-compartment culture dishes were prepared by placing cloning cylinders ( $8 \mathrm{~mm}$ diameter; Bellco Glass, Inc., Vineland, NJ), constituting the inner compartment, in the center of 35 $\mathrm{mm}$ culture dishes (Corning Costar, Cambridge, MA). The cylinders were kept in place with high-vacuum silicon grease moistened with $6 \%$ methylcellulose in culture medium. A schematic drawing of the compartmented model has been published previously (Vikman et al., 2001).

Spinal cords and DRGs were dissected from Sprague Dawley rats at embryonic day 17 (B\&K Universal, Sollentuna, Sweden). Dissociated DRG cells were seeded $\left(1.4 \times 10^{5}\right.$ cells/dish $)$ into the inner compartment. The dorsal thirds of the spinal cords were incubated in $0.1 \%$ trypsin for $20 \mathrm{~min}$ at $37^{\circ} \mathrm{C}$, followed by dissociation in culture medium. The dorsal horn cells were seeded into the outer compartment at a density of $5 \times 10^{5}$ cells/dish. The cultures were maintained at $37^{\circ} \mathrm{C}$ and $5 \% \mathrm{CO}_{2}$ and used for experiments after $17 \mathrm{~d}$ in culture.

Electrophysiology. Whole-cell patch-clamp recordings and electrical stimulation were performed as described by Vikman et al. (2001). The dorsal horn neurons were kept at resting potential during current-clamp recording unless otherwise stated. For electrical stimulation of the DRG axons, a stimulator (model 2100; A-M Systems, Inc., Carlsborg, WA) with its two output poles connected to Ag wires was used. One wire was placed into the solution of the inner compartment and the other into the solution of the outer compartment, each $\sim 2-3 \mathrm{~mm}$ on either side of the inner compartment wall. This arrangement allowed stimulation of DRG axons projecting underneath the inner compartment wall, which in turn gave rise to synaptic responses in the dorsal horn neuronal network. The stimulus threshold (i.e., the least amount of current needed to evoke a change in postsynaptic activity in the dorsal horn neuronal network) varied between the recordings, ranging from 100 to $600 \mu \mathrm{A}$. The stimulus intensity during LFS and HFS was increased to $\sim 1.5 \times$ threshold to ensure an efficient stimulation of the DRG axons. In addition, a pulse duration of 2-5 msec was used in all experiments because this has been observed previously to trigger an optimal stimuli-evoked response in the DRG axon population (Vikman et al., 2001). For LFS experiments, cultures were repetitively stimulated at a defined frequency. Several different frequencies $(1-10 \mathrm{~Hz})$ were sometimes tested for each dorsal horn neuron recorded, but the frequency was always held constant during a stimulus train. For HFS experiments, a series $(n=6-9)$ of test stimuli (TSs) (1 or $10 \mathrm{~Hz} ; 1 \mathrm{sec}$ train duration) at threshold intensity were applied at various time intervals $(>30 \mathrm{sec})$ to define the pre-HFS response to TSs. This was followed by a series of four to five trains of HFS $(100 \mathrm{~Hz} ; 1-2 \mathrm{sec}$ train duration; $1.5 \times$ threshold intensity) at $10 \mathrm{sec}$ intervals. The clamped neuron was allowed to rest for 2-3 min after the HFS series had been completed before a new series of TSs was given to determine the post-HFS response.

Experiments were performed on cultured neurons from three different cell culture preparations. During LFS experiments, recordings from two to three dorsal horn neurons were performed for each cell culture dish. For HFS experiments, however, recordings were performed only from one neuron per dish to avoid the interaction of possible long-lasting effects from previous recordings.

Data analyses. All data were analyzed using Clampfit software (Axon Instruments, Union City, CA). For LFS experiments, the number of cells responding to repetitive stimulation with cumulative depolarization, increased action potential (AP) frequency, or hyperpolarization was counted and compared with the total number of cells tested. The peak depolarization and relative time to peak were calculated for analysis of frequency dependency. For HFS experiments, the number of TS-evoked APs was counted for each TS, both before HFS and after HFS, which is similar to the parameter used for analysis of LTP by others (Svendsen et al., 1999a). Statistical differences in the number of evoked APs after HFS compared with before HFS, as well as differences in membrane potential between neuronal populations, were assessed via a Student's $t$ test using Microsoft Excel software (Microsoft, Seattle, WA). Data were prepared for illustration with CorelDraw (Corel Corp., Ottawa, Ontario, Canada) and Adobe software (Adobe Systems, San Jose, CA). Data are presented as mean $\pm \mathrm{SEM}$ in Results.

\section{RESULTS}

Current-clamp recordings of synaptic activity were performed on dorsal horn neurons in the outer compartment. All recorded neurons exhibited robust spontaneous activity, both excitatory and inhibitory. To evaluate the responsiveness and stimulus threshold of the dorsal horn neurons to DRG axon stimulation, single test pulses were given to the culture at various time intervals. Neurons with a clear response to stimulation, consisting of APs and/or EPSPs, were chosen for experiments.

\section{LFS-mediated sensitization of dorsal horn neurons}

The response to repetitive LFS with constant frequency was recorded from 17 dorsal horn neurons. In 10 of the 17 cells (59\%), the repetitive LFS resulted in a cumulative depolarization, which lasted throughout the pulse train without returning to baseline between pulses. The depolarization slowly decayed back to resting level after the end of the stimulus train. In 7 of the 10 cells that responded with cumulative depolarization (41\% of the total number of cells), a marked increase in AP and/or EPSP frequency could be observed during the pulse train (Fig. 1 $A$ ). In some neurons this increased spike frequency ceased directly at the end of the stimulus train, but in others, as seen in Figure $1 \mathrm{~A}$, the enhanced firing rate continued as an afterdischarge, before returning to baseline level. In all seven neurons, the LFSmediated response was followed by a prolonged potentiation of spontaneous synaptic activity compared with that observed before application of LFS.

In the remaining 7 of the 17 dorsal horn neurons tested, repetitive stimulation at a constant frequency did not evoke a depolarization plateau or increased AP frequency. Although these cells clearly responded to each stimulus pulse, no cumulative depolarization was generated. These seven neurons all exhibited a marked amount of inhibitory spontaneous activity. In five of the seven neurons, the membrane potential decayed back to baseline level between stimulus pulses, but in two cells, each pulse resulted in a small hyperpolarization, which summed to create a prolonged hyperpolarizing response (Fig. 1B). This hyperpolarization gradually returned to resting potential after the end of the stimulus train.

The LFS-induced sensitization of dorsal horn neurons tended to be frequency dependent (Fig. 1A,C). This varied somewhat between the different neurons analyzed, but in general, frequencies of $<1 \mathrm{~Hz}$ and $>10 \mathrm{~Hz}$ did not evoke any cumulative depolarization or increased firing rate. Although variations in responsiveness were observed between neurons, the frequency dependency was always constant for each neuron tested and showed a similar response pattern to repeated application of LFS of the same magnitude. A common feature for those neurons in which a cumulative depolarization was induced without any marked change in AP frequency was that the peak value of the depolarization plateau increased with increased stimulus fre- 
A

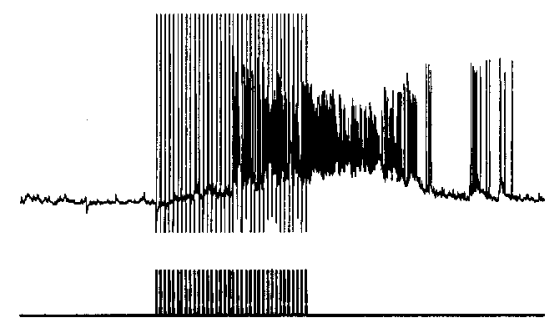

C

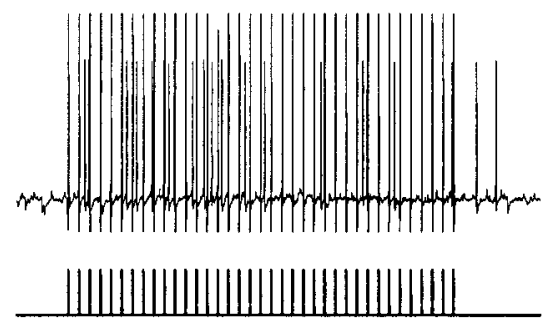

$50 \mathrm{mV}$
B

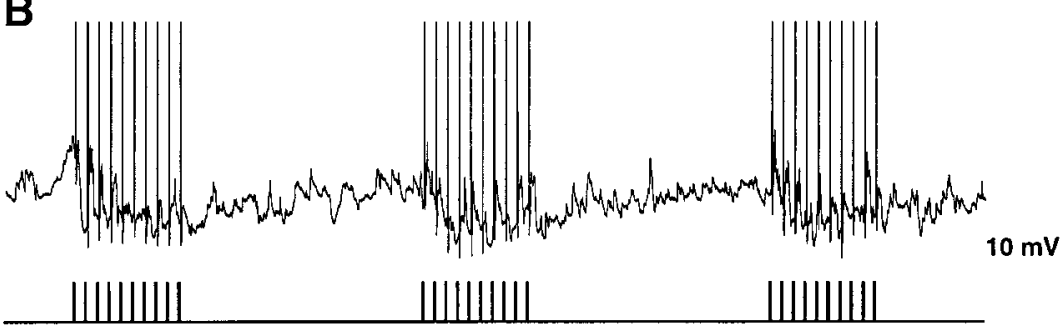

$5 \mathrm{~s}$

$1 \mathrm{~s}$
Figure 1. LFS-induced responses in cultured dorsal horn neurons. $A$, Repetitive LFS at $5 \mathrm{~Hz}$ $(500 \mu \mathrm{A} ; 5 \mathrm{msec})$ resulting in cumulative depolarization and a concomitant burst of APs in a dorsal horn neuron. A marked afterdischarge is seen after the end of the LFS. $B$, Repetitive, short-pulse trains $(10 \mathrm{~Hz} ; 1 \mathrm{sec}$ duration; 400 $\mu \mathrm{A} ; 5 \mathrm{msec}$ ) resulted in a hyperpolarizing response in a subset of neurons. $C$, Same neuron as in $A$, responding to LFS at $2 \mathrm{~Hz}$. No marked cumulative depolarization can be seen and only a slight increase in firing frequency during the pulse train is noted. The bottom traces in $A-C$ represent output from the stimulator. quency (i.e., in this case from $2.3 \pm 0.5 \mathrm{mV}$ to $8.7 \pm 1.8 \mathrm{mV}$ when the frequency was raised from 2 to $5 \mathrm{~Hz}$ ) (Fig. $2 A$ ). On the other hand, when the LFS evoked a burst of APs during the pulse train, the peak depolarization was markedly greater $(21.1 \pm 0.8 \mathrm{mV})$ and fairly constant for the given frequencies (Fig. $2 A$ ). However, a frequency dependency also was noted in this latter case because the relative time from the first pulse in the stimulus train to the peak depolarization of the AP burst decreased with increased frequency (Fig. $2 B$ ). When the frequency was increased to $>10$ $\mathrm{Hz}$ for these cells, no AP burst could be induced (data not shown).

\section{HFS-induced potentiation of dorsal horn neuronal responsiveness}

Changes in excitability after repetitive HFS were studied in 12 dorsal horn neurons. The number of APs in response to TSs was compared before and after HFS. The pre-HFS response varied in appearance between neurons; in some the TS evoked one or several APs in combination with EPSPs, whereas in others a burst consisting only of EPSPs occurred. However, the response was more or less constant for each TS before HFS for a given neuron. To avoid summation of postsynaptic responses, the TSs were separated by at least $30 \mathrm{sec}$. In 5 of the 12 cells (42\%), the HFS induced a significant increase $(p \leq 0.05)$ in neuronal responsiveness (i.e., number of spikes per TS). The HFS-induced potentiation of the response to sequentially administered TSs for one cell is illustrated in Figure $3 A$. Examples of the response to one TS before HFS (Fig. 3A, left trace) and one TS after HFS (Fig. 3A, right trace) are shown below the graph. One additional neuron showed potentiated responsiveness after HFS, but the increase in number of spikes was not significant $(p=0.11)$. In four of the six HFS-responding neurons, the potentiation outlasted the recording time ( $1 \mathrm{hr}$ maximum), whereas in the other two cells, the response began to decay after $\sim 15 \mathrm{~min}$. However, this response did not fully return to baseline level before the end of the recording period. In the remaining six cells, no alteration in response to TSs after HFS was observed (Fig. 2B).

In contrast to the HFS-induced potentiation described above,
A

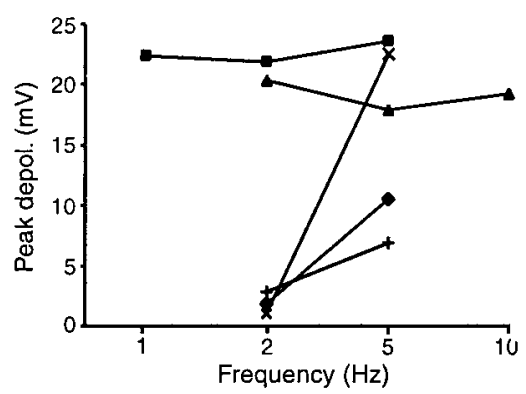

B

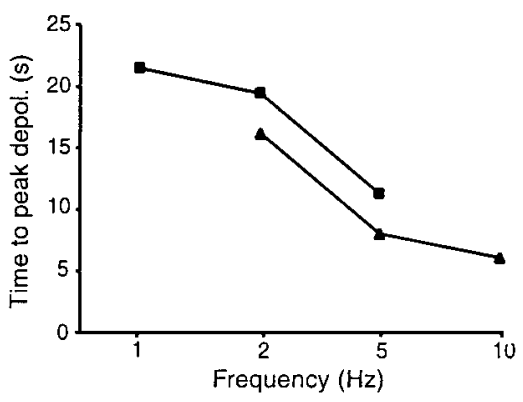

Figure 2. Frequency-dependent changes in dorsal horn neuronal responsiveness to LFS. A, Graph illustrating the response of five different neurons $(\boldsymbol{\square}, \boldsymbol{\vee}, \times, \boldsymbol{\Delta}$, and + ) to repetitive LFS at $1,2,5$, and $10 \mathrm{~Hz}$. An increase in stimulus frequency from 2 to $5 \mathrm{~Hz}$ resulted in a greater peak depolarization for those neurons that responded to LFS with a cumulative depolarization only $(\bullet,+)$. When LFS induced a burst of APs during the stimulus train, the peak depolarization was markedly increased $(\times, \mathbf{\square}, \mathbf{\Delta})$ and did not vary with frequency $(\boldsymbol{\square}, \mathbf{\Delta})$. Note that $\times$ responds to $2 \mathrm{~Hz}$ stimulation with a cumulative depolarization only, whereas $5 \mathrm{~Hz}$ stimulation evokes an AP burst. $B$, Graph illustrating the frequency dependency of the relative time from start of LFS to peak depolarization for the neurons responding with a burst of APs. $\boldsymbol{\square}$ and $\boldsymbol{\Delta}$ illustrate the same neurons as in $A$. Increased frequency results in decreased latency. Lack of response for the cells at different frequencies is not shown in the graphs. 
A

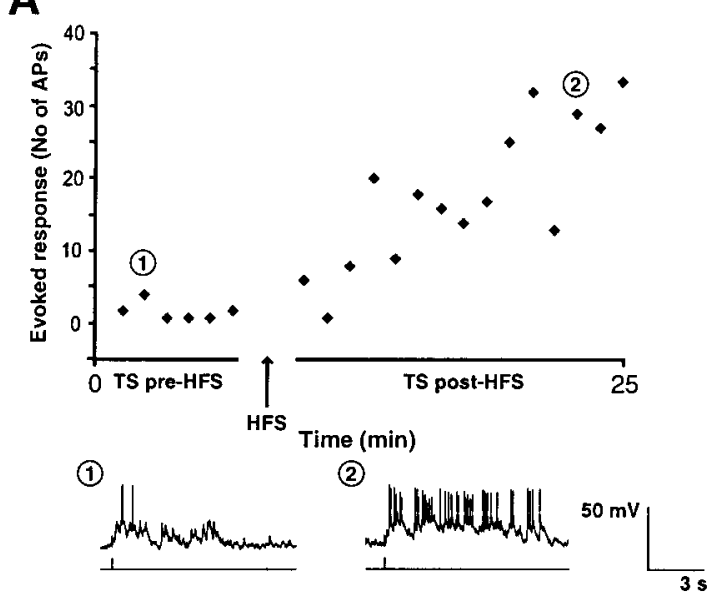

C

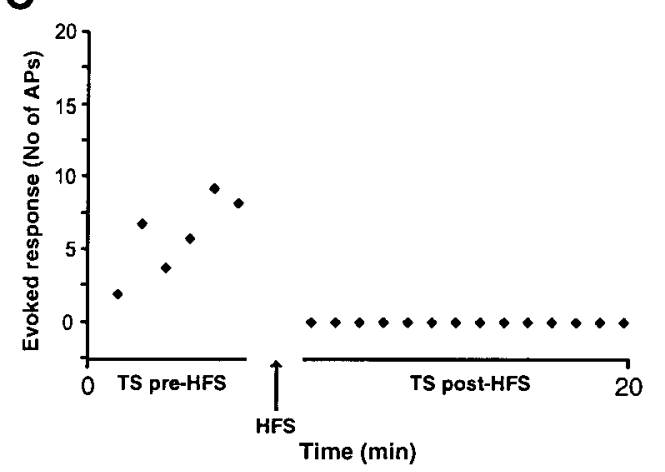

B

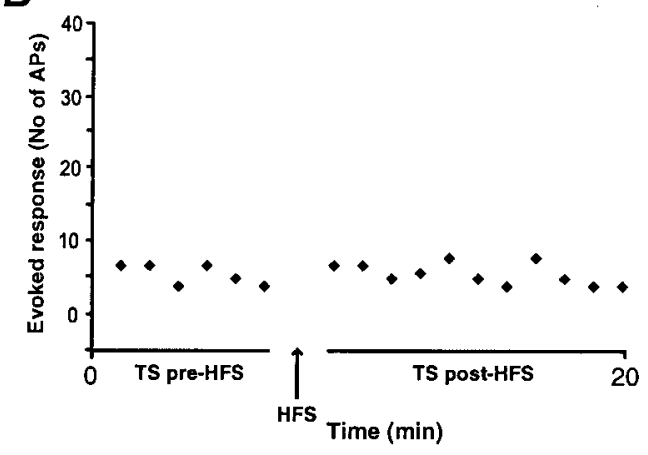

D

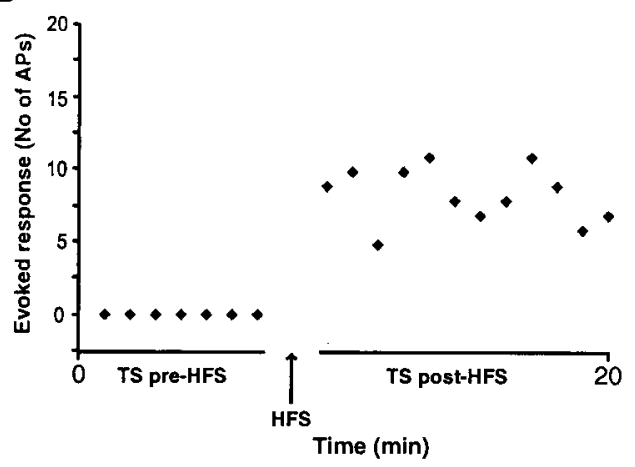

Figure 3. HFS induced alteration of dorsal horn neuronal excitability in an LTP-like manner. The number of evoked APs as a response to sequentially administered TSs $(\checkmark)$ is shown. $A$, Graph illustrating the increase in responsiveness for one dorsal horn neuron after tetanic stimulation (100 $\mathrm{Hz}$; 600 $\mu \mathrm{A} ; 5 \mathrm{msec})$. The traces marked 1 and 2 below the graph correspond to the TS $(1 \mathrm{~Hz} ; 400 \mu \mathrm{A} ; 5 \mathrm{msec})$ pre-HFS and post-HFS marked 1 and 2 , respectively, in the graph. Note the increased number of APs after HFS. B, Graph illustrating the lack of a HFS-induced effect in one neuron. No significant alteration in the number of TS-evoked APs is seen. $C, D$, Graphs illustrating the influence of the postsynaptic membrane potential on the HFS-induced effect observed in a subset of neurons tested. $C$, Tetanic stimulation $(100 \mathrm{~Hz} ; 600 \mu \mathrm{A} ; 5 \mathrm{msec})$ at resting potential resulted in a depression of the TS-evoked response. $D$, The same neuron as in $C$, but the membrane potential for this neuron was slightly depolarized during HFS, which instead mediated a potentiation of the response and thereby reversed the previously induced depression. Arrows indicate application of a series of four to five HFS trains.

application of HFS at resting potential in three of the neurons tested resulted in a depression of synaptic activity (i.e., a decreased number of spikes per TS) (Fig. $3 C$ ). To test whether the HFS-induced modification of synaptic activity was dependent on the membrane potential of the postsynaptic neuron, as reported previously for dorsal horn neurons in vitro (Randic et al., 1993), an additional set of HFS was applied when the membrane potential of the recorded neuron was slightly depolarized $(8.3 \pm 1.3$ $\mathrm{mV})$. This resulted in a potentiation of the synaptic response similar to that described above in all three neurons (Fig. 3D). The average membrane potential for these three neurons did not differ significantly from that for the other nine neurons in the experimental group $\left(V_{\mathrm{m}}=61.3 \pm 1.8\right.$ and $V_{\mathrm{m}}=65.3 \pm 1.9$, respectively; $p=0.2$ ) or from the average membrane potential for the entire experimental group $\left(V_{\mathrm{m}}=64.3 \pm 1.6 ; p=0.3\right)$.

\section{DISCUSSION}

\section{The LFS-induced increase in AP frequency shows} characteristics of classical wind-up

The observed increase in AP frequency during LFS in our system shares characteristics with the previously described wind-up in the nociceptive system (Herrero et al., 2000). Wind-up of dorsal horn neuronal responsiveness was originally described during repetitive LFS of C-fibers in the cat sural nerve (Mendell and Wall, 1965). The phenomenon has since then been observed in several experimental animal species and it can, under normal conditions, only be evoked by stimulation of $\mathrm{C}$-fibers. Thus, the finding that wind-up can be induced in our culture system provides additional strong evidence for the occurrence of functional synaptic connections between $\mathrm{C}$-fibers and dorsal horn neurons in this model (Vikman et al., 2001).

As mentioned in the introductory remarks, wind-up is a frequency-dependent phenomenon (i.e., it generally does not occur at $<0.2-0.3 \mathrm{~Hz}$, reaches a maximum at $1-2 \mathrm{~Hz}$, and declines at $>20 \mathrm{~Hz}$ ) (Schouenborg, 1984). In accordance with this, we obtained evidence of frequency dependency in our experiments. A feature observed in our model, and which has been described in connection with wind-up, is a slow accumulating depolarization of the membrane potential seen during the stimulus train (Sivilotti et al., 1993; Jeftinija and Urban, 1994). Cumulative depolarization has been suggested to predict the probability of appearance of wind-up (Sivilotti et al., 1993). In addition, the typical afterdischarge of APs that follows wind-up was also seen in our model. Another observation was the cumu- 
lative hyperpolarizing response in some of the "wind-upnegative" cells, which is similar to that reported by Jeftinija and Urban (1994). Together, these findings indicate that the wind-up observed in the dispersed dorsal horn neurons has the same characteristics as that seen in preparations of spinal cord with intact structures.

\section{Does the observed HFS-induced potentiation reflect LTP?}

In addition to LFS-mediated AP wind-up, we also observed HFS-induced potentiation of neuronal responsiveness in the cultures, in which the response to TS was increased after application of a series of HFS (i.e., tetanic stimulation). Evidence that tetanic stimulation can induce changes in synaptic activity, such as LTP and long-term depression (LTD), in the dorsal horn was first described by Randic et al. (1993). During recent years, several groups have presented evidence for induction of LTP in dorsal horn neurons after electrical or noxious stimulation and after nerve injury (Svendsen et al., 1997; Sandkühler and Liu, 1998). HFS may also induce "post-tetanic potentiation" (PTP), which resembles LTP but is of shorter duration (minutes to hours); LTP can last from hours to days (Fisher et al., 1997). PTP has been suggested as a predictor of LTP (Son and Carpenter, 1996), but is thought to be the result primarily of presynaptic mechanisms, whereas LTP is attributable predominantly to postsynaptic changes (Fisher et al., 1997; Malinow et al., 2000). Establishing whether our observations are equivalent to PTP or LTP is hampered by the fact that whole-cell recording in cultured cells cannot be maintained for $>1$ or $2 \mathrm{hr}$, thus making it difficult to distinguish between these phenomena by a time factor. However, in a subset of the cells recorded, we found that the membrane potential of the postsynaptic cell more or less determined whether the HFS-induced response was potentiated or suppressed in a manner similar to that described previously for LTP and LTD (Randic et al., 1993; Liu et al., 1998). This would indicate that the HFS-induced potentiation observed in our culture model is not only attributable to an increased transmitter release from the presynaptic terminals, as in PTP, but may also rely on postsynaptic changes, indicative of LTP rather than PTP.

\section{Relevance of wind-up and LTP for studies of central sensitization}

Both wind-up and LTP have been discussed in relation to the generation of postinjury pain hypersensitivity (Baranauskas and Nistri, 1998). Wind-up is not necessarily equivalent to central sensitization (Woolf, 1996), and whether wind-up is appropriate as a model for studies of the mechanisms of sensitization has therefore been a matter of debate (Svendsen et al., 1999c; Dickenson et al., 2000). However, a recent study by Li et al. (1999) shows that wind-up leads not only to an increase in the neuronal responses to $\mathrm{C}$-fiber stimulation but also to other characteristics of central sensitization, such as an expanded receptive field, and may therefore, even if not equivalent to central sensitization, be considered as a useful and convenient tool for studying this phenomenon.

Whether LTP in the dorsal horn is of any pathophysiological relevance has also been questioned, because the probability of tetanic stimulation occurring naturally is not very high (Paulsen and Sejnowski, 2000). Recently, however, several reports have demonstrated LTP in the spinal dorsal horn of intact animals (Liu and Sandkühler, 1997; Svendsen et al., 1999b) and that it can be induced by natural noxious stimulation (Rygh et al., 1999). The idea that long-lasting changes in synaptic plasticity, such as LTP, may contribute to the development of central sensitization is supported by the finding that generation of neuropathic pain-related behavior in rats was accompanied by LTP-like changes in dorsal horn excitability (Miletic and Miletic, 2000; Draganic et al., 2001).

The findings of wind-up and LTP-like responses in our cell culture model make the system useful for detailed studies, at the synaptic and cellular level, of the relationship between the biophysical properties of the neurons and their ability to develop hypersensitivity. In addition, direct correlative studies of changes in synaptic protein composition and gene expression after hyperexcitability are also possible because the neurons are easily distinguishable in the system. In recent years, glial cells have become more prominent in the field of synaptic plasticity (for review, see Haydon, 2001). An interesting aspect would therefore be to study the possible influence of glial cells on central sensitization. Because glial cells are present at both the peripheral (DRG) and central (dorsal horn) level in our compartmented culture system, the model not only creates an in vivo-like milieu, with neuronal and glial elements growing together, but also provides a convenient means for such studies.

\section{REFERENCES}

Baranauskas G, Nistri A (1998) Sensitization of pain pathways in the spinal cord: cellular mechanisms. Prog Neurobiol 54:349-365.

Dickenson AH, Stanfa LC, Kontinen V, Suzuki R, Carpenter K (2000) Comment on: Svendsen et al; some problems with wind-up and its calculation, PAIN 83 (1999) 109-111. Pain 85:524.

Draganic P, Miletic G, Miletic V (2001) Changes in post-tetanic potentiation of A-fiber dorsal horn field potentials parallel the development and disappearance of neuropathic pain after sciatic nerve ligation in rats. Neurosci Lett 301:127-130.

Fisher SA, Fischer TM, Carew TJ (1997) Multiple overlapping processes underlying short-term synaptic enhancement. Trends Neurosci 20:170-177.

Haydon PG (2001) GLIA: listening and talking to the synapse. Nat Rev Neurosci 2:185-193.

Herrero JF, Laird JM, Lopez-Garcia JA (2000) Wind-up of spinal cord neurones and pain sensation: much ado about something? Prog Neurobiol 61:169-203.

Jeftinija S, Urban L (1994) Repetitive stimulation induced potentiation of excitatory transmission in the rat dorsal horn: an in vitro study. J Neurophysiol 71:216-228.

Li J, Simone DA, Larson AA (1999) Windup leads to characteristics of central sensitization. Pain 79:75-82.

Liu X, Sandkühler J (1997) Characterization of long-term potentiation of C-fiber-evoked potentials in spinal dorsal horn of adult rat: essential role of NK1 and NK2 receptors. J Neurophysiol 78:1973-1982.

Liu XG, Morton CR, Azkue JJ, Zimmermann M, Sandkühler J (1998) Long-term depression of C-fibre-evoked spinal field potentials by stimulation of primary afferent $\mathrm{A} \delta$-fibres in the adult rat. Eur $\mathrm{J}$ Neurosci 10:3069-3075.

Malinow R, Mainen ZF, Hayashi Y (2000) LTP mechanisms: from silence to four-lane traffic. Curr Opin Neurobiol 10:352-357.

Mendell LM, Wall PD (1965) Responses of single dorsal cord cells to peripheral cutaneous unmyelinated fibres. Nature 206:97-99.

Miletic G, Miletic V (2000) Long-term changes in sciatic-evoked A-fiber dorsal horn field potentials accompany loose ligation of the sciatic nerve in rats. Pain 84:353-359.

Paulsen O, Sejnowski TJ (2000) Natural patterns of activity and longterm synaptic plasticity. Curr Opin Neurobiol 10:172-179.

Randic M, Jiang MC, Cerne R (1993) Long-term potentiation and longterm depression of primary afferent neurotransmission in the rat spinal cord. J Neurosci 13:5228-5241.

Rygh LJ, Svendsen F, Hole K, Tjølsen A (1999) Natural noxious stimulation can induce long-term increase of spinal nociceptive responses. Pain 82:305-310.

Sandkühler J, Liu X (1998) Induction of long-term potentiation at spinal synapses by noxious stimulation or nerve injury. Eur $\mathrm{J}$ Neurosci 10:2476-2480.

Schouenborg J (1984) Functional and topographical properties of field potentials evoked in rat dorsal horn by cutaneous $\mathrm{C}$-fibre stimulation. J Physiol (Lond) 356:169-192.

Sivilotti LG, Thompson SW, Woolf CJ (1993) Rate of rise of the cumu- 
lative depolarization evoked by repetitive stimulation of small-caliber afferents is a predictor of action potential windup in rat spinal neurons in vitro. J Neurophysiol 69:1621-1631.

Son H, Carpenter DO (1996) Interactions among paired-pulse facilitation and post-tetanic and long-term potentiation in the mossy fiber-CA3 pathway in rat hippocampus. Synapse 23:302-311.

Svendsen F, Tjølsen A, Hole K (1997) LTP of spinal A $\beta$ and C-fibre evoked responses after electrical sciatic nerve stimulation. NeuroReport 8:3427-3430

Svendsen F, Tjølsen A, Rygh LJ, Hole K (1999a) Expression of longterm potentiation in single wide dynamic range neurons in the rat is sensitive to blockade of glutamate receptors. Neurosci Lett 259:25-28.
Svendsen F, Tjølsen A, Gjerstad J, Hole K (1999b) Long term potentiation of single WDR neurons in spinalized rats. Brain Res 816:487-492.

Svendsen F, Hole K, Tjølsen A (1999c) Some problems with wind-up and its calculation. Pain 83:109-112.

Vikman KS, Backström E, Kristensson K, Hill RH (2001) A twocompartment in vitro model for studies of modulation of nociceptive transmission. J Neurosci Methods 105:175-184.

Woolf CJ (1983) Evidence for a central component of post-injury pain hypersensitivity. Nature 306:686-688.

Woolf CJ (1996) Windup and central sensitization are not equivalent. Pain 66:105-108. 